/* Aggregation Approximation */

\#include $<$ stdio.h $>$

\#include $<$ stdlib.h $>$

\#include $<$ math.h $>$

\#define N 10000 /* Detection size *

\#define M $100 / *$ Aggregation size */

\#define $\lambda 2.0 / *$ Parameter: division rate (sensitive) */

\#define $\mu 1.0 / *$ Parameter: death rate (sensitive) $* /$

\#define $\alpha 3.0 / *$ Parameter: division rate (resistant) $* /$

\#define $\beta 1.0 / *$ Parameter: death rate (resistant) */

\#define $\gamma 0.00001 / *$ Parameter: mutation rate */

int num,i,j;

double **dmatrix(int nr1, int nr2, int nl1, int n12); /* Memory allocation */

void free_dmatrix(double **a, int nr1, int nr2, int nl1, int nl2); /* Memory release */

void up_matrix (double **a, char c, FILE * fout); /* Function for calculating matrix (exact): $\mathrm{P}_{\mathrm{i}} * /$

void dn matrix (double **b, char c, FILE *fout); $/ *$ Function for calculating matrix (exact): $\mathrm{Q}_{\mathrm{i}} *$

void en matrix (double **e, char c, FILE *fout); /* Function for calculating unit matrix (exact): $\mathrm{I}_{\mathrm{i}} * /$

void product1 (double **c, double **b, double **f, double **e, double **sa, FILE *fout); / Function for calculating (exact): $\mathrm{I}_{\mathrm{i}+1}-\mathrm{Q}_{\mathrm{i}} \mathrm{F}_{\mathrm{i}-1} * /$

void gauss (double **sa, double **inv sa, FILE *fout); /* Function for calculating (exact): $\left(\mathrm{I}_{\mathrm{i}+1}-\mathrm{Q}_{\mathrm{i}} \mathrm{F}_{\mathrm{i}-1}\right)^{-1} * /$

void product2 (double **inv_sa, double **a, double **g, FILE *fout); /* Function for calculating $\left(\right.$ exact) $: \mathrm{F}_{\mathrm{i}}=\left(\mathrm{I}_{\mathrm{i}+1}-\mathrm{Q}_{\mathrm{i}} \mathrm{F}_{\mathrm{i}-1}\right)^{-1} \mathrm{P}_{\mathrm{i}} * /$

void product3 (double $* *$ d, double $* * D$, double $* * g$, FILE $*$ fout); $/ *$ Function for calculating (exact) $: \pi_{i+1}=(1,0) \mathrm{F}_{1} \mathrm{~F}_{2} \mathrm{~F}_{3} \ldots \mathrm{F}_{\mathrm{i}-1} \mathrm{~F}_{\mathrm{i}} * /$

void product4 (double ${ }^{* *}$ dinit, double ${ }^{* *} \mathrm{~g}$, double ${ }^{* *} \mathrm{~d}$, FILE $*$ fout); $/ *$ Function for calculating $\left(\right.$ exact) $: \pi_{2}=(1,0) \mathrm{F}_{1} * /$

void upabs matrix (double **a abs, char c, FILE *fout); /* Function for calculating matrix (aggregation) : $\mathrm{P}_{\mathrm{i}} * /$

void dnabs_matrix (double **b_abs, char c, FILE *fout); /* Function for calculating matrix (aggregation) : $\mathrm{Q}_{i} * /$

void enabs matrix (double **e abs, char c, FILE *fout); /* Function for calculating matrix (aggregation) : $\mathrm{I}_{\mathrm{i}} * /$

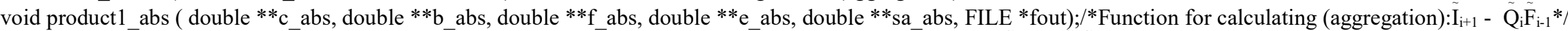

void gauss_abs( double **sa_abs, double **inv_saabs,FILE *fout); /*Function for calculating (aggregation): $\left(\tilde{\mathrm{I}}_{\mathrm{i}+1}-\tilde{\mathrm{Q}}_{\mathrm{i}} \tilde{\mathrm{F}}_{\mathrm{i}-1}\right)^{-1} * /$ 
void product2_abs( double **inv_saabs, double **a_abs,double **g_abs,FILE *fout); /* Function for calculating (aggregation) : $\tilde{\mathrm{F}}_{\mathrm{i}}=\left(\tilde{\mathrm{I}}_{\mathrm{i}+1}-\tilde{\mathrm{Q}}_{\mathrm{i}} \tilde{\mathrm{F}}_{\mathrm{i}-1}\right)^{-1} \tilde{\mathrm{P}}_{\mathrm{i}}{ }^{*} /$ void product3_abs(double $* *$ d_abs, double **D_abs, double **g_abs,FILE *fout); / Function for calculating $($ aggregation $): \tilde{\pi}_{\mathrm{i}+1}=(1,0) \tilde{\mathrm{F}}_{1} \tilde{\mathrm{F}}_{2} \tilde{\mathrm{F}}_{3} \ldots \tilde{\mathrm{F}}_{\mathrm{i}-1} \tilde{\mathrm{F}}_{\mathrm{i}} * /$

void pre_product2(double **inv_sa, double **a_abs,double **g_abs,FILE *fout); ${ }^{*}$ Function for calculating (aggregation point) : $\tilde{\mathrm{F}}_{\mathrm{i}}=\left(\tilde{\mathrm{I}}_{\mathrm{i}+1}-\tilde{\mathrm{Q}}_{\mathrm{i}} \tilde{\mathrm{F}}_{\mathrm{i}-1}\right)^{-1} \tilde{\mathrm{P}}_{\mathrm{i}} * /$ void pre_product3( double $* *$ d_abs, double $* * D$, double $* * g \_a b s, F I L E *$ fout); $/ *$ Function for calculating (aggregation point) : $\pi_{i+1}=(1,0) F_{1} F_{2} F_{3} \ldots F_{i-1} F_{i} * /$ double keisan (double **x, double *prob, double *prob_d, char c, FILE *fout); /* Function for calculating probability */

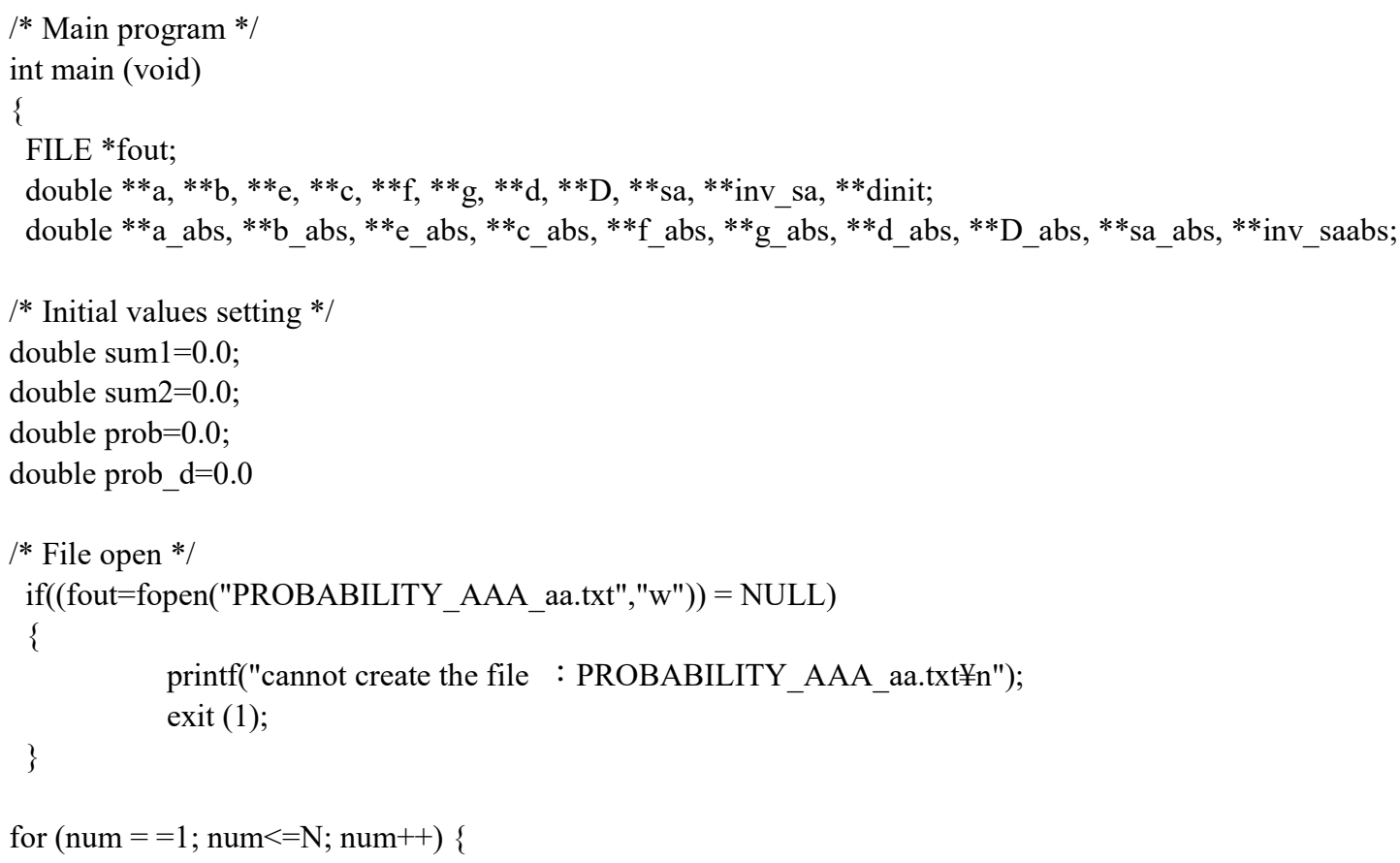

* Calculation: $\pi_{2}$ and emerging probability of resistance */ if $($ num $==1)$

/* Memory allocation */ 
$\mathrm{a}=\operatorname{dmatrix}(1, \operatorname{num}+1,1, \operatorname{num}+2)$;

$\mathrm{g}=\operatorname{dmatrix}(1$, num $+1,1$, num +2$)$;

$\mathrm{d}=\operatorname{dmatrix}(1,1,1$, num +2$)$

dinit $=$ dmatrix $(1,1,1,2)$;

$/ * \mathrm{P}_{\mathrm{i}} *$

up_matrix(a,'G',fout);

for $(\mathrm{i}=1 ; \mathrm{i}<=$ num $+1 ; \mathrm{i}++)$ ?

$$
\begin{aligned}
& \text { for }(j=1 ; j<=n u m+2 ; j++)\{ \\
& g[i][j]=a[i][j] ;
\end{aligned}
$$

\}

*Calculation: $\pi_{2}=(1,0) \mathrm{F}_{1} * /$

product4(dinit, $g, d$, fout);

* Probability of resistance */

keisan(dint, prob, prob_d, P, *fout);

/* Print out */

fprintf (fout,"Probability is: ¥n",c);

fprintf (fout,"\%12.10草", keisan(d,'P', fout));

fprintf (fout,"¥n");

/* Memory release */

free dmatrix (a, 1, num $+1,1$, num +2 );

free_dmatrix(dinit, 1, 1, 1, 2);

\}

/* Calculation Before Aggregation (= exact analysis) */

else if (num $>=2 \& \&$ num $<=M$ )

\{

/* Memory allocation */ 
$\mathrm{f}=\operatorname{dmatrix}(1$, num, 1, num +1$) ;$
$\mathrm{D}=\operatorname{dmatrix}(1,1,1$, num +1$) ;$

for $(\mathrm{i}=1 ; \mathrm{i}<=$ num; $\mathrm{i}++)\{$

for $(j=1 ; j<=$ num $+1 ; j++)\{$

$\mathrm{f}[\mathrm{i}][\mathrm{j}]=\mathrm{g}[\mathrm{i}][\mathrm{j}]$;

\}

$$
\}
$$

for $(\mathrm{i}=1 ; \mathrm{i}<=1 ; \mathrm{i}++)\{$

for $(\mathrm{j}=1 ; \mathrm{j}<=$ num $+1 ; \mathrm{j}++)\{$

$\mathrm{D}[\mathrm{i}][\mathrm{j}]=\mathrm{d}[\mathrm{i}][\mathrm{j}]$;

\}

/* Memory release */

free_dmatrix (g, 1, num, 1, num +1$)$;

free_dmatrix (d, 1, 1, 1, num +1$)$;

/* Memory allocation */

$\mathrm{a}=\operatorname{dmatrix}(1$, num $+1,1$, num +2$)$;

$\mathrm{b}=$ dmatrix $(1$, num $+1,1$, num $)$;

$\mathrm{c}=$ dmatrix $(1$, num $+1,1$, num +1$)$;

$\mathrm{e}=$ dmatrix $(1$, num $+1,1$, num +1$)$;

$\mathrm{g}=$ dmatrix $(1$, num $+1,1$, num +2$)$;

$\mathrm{sa}=\operatorname{dmatrix}(1$, num $+1,1$, num +1$) ;$

inv sa $=\operatorname{dmatrix}(1$, num $+1,1$, num +1$)$;

$\mathrm{d}=\overline{\operatorname{dmatrix}}(1,1,1$, num +2$)$;

/* Matrix setting: $\mathrm{P}_{\mathrm{i}}, \mathrm{Q}_{\mathrm{i}}, \mathrm{I}_{\mathrm{i}}$ */

up_matrix(a,'A',fout);

dn matrix (b,'B',fout);

en_matrix(e,'I',fout);

/* Calculation: $\pi_{\mathrm{i}+1}=(1,0) \mathrm{F}_{1} \mathrm{~F}_{2} \mathrm{~F}_{3} \ldots \mathrm{F}_{\mathrm{i}-1} \mathrm{~F}_{\mathrm{i}} * /$

product1(c,b,f,e,sa,fout); /* $\mathrm{I}_{\mathrm{i}+1}-\mathrm{Q}_{\mathrm{i}} \mathrm{F}_{\mathrm{i}-1} * /$ 
gauss(sa,inv sa,fout); $/ *\left(\mathrm{I}_{\mathrm{i}+1}-\mathrm{Q}_{\mathrm{i}} \mathrm{F}_{\mathrm{i}-1}\right)^{-1} * /$

product2(inv_sa,a,g,fout); $/ * \mathrm{~F}_{\mathrm{i}}=\left(\mathrm{I}_{\mathrm{i}+1}-\mathrm{Q}_{\mathrm{i}} \mathrm{F}_{\mathrm{i}-1}\right)^{-1} \mathrm{P}_{\mathrm{i}} * /$

product3(d,D,g,fout); $\quad / * \pi_{\mathrm{i}+1}=(1,0) \mathrm{F}_{1} \mathrm{~F}_{2} \mathrm{~F}_{3} \ldots \mathrm{F}_{\mathrm{i}-1} \mathrm{~F}_{\mathrm{i}} * /$

/ Probability of resistance */

keisan(d_abs, prob, prob_d, P, *fout);

/* Print out */

fprintf( fout,"Probability is : ¥n",c);

fprintf( fout,"\%12.10f¥t",keisan(d,'P', fout));

fprintf( fout,"¥n");

/* Memory release */

free_dmatrix (a, 1, num $+1,1$, num +2$)$;

free dmatrix (b, 1 , num $+1,1$, num);

free_dmatrix (c, 1, num $+1,1$, num +1$)$;

free dmatrix (e, 1, num $+1,1$, num +1$)$;

free_dmatrix (f, 1 , num, 1 , num +1 );

free_dmatrix (sa, 1, num $+1,1$, num +1$)$;

free dmatrix (inv sa, 1 , num $+1,1$, num +1 );

free_dmatrix (D, 1, 1, 1, num +1$)$;

* Calculation Aggregation Point */

else if (num $==M+1$ )

/* Memory allocation */

$\mathrm{f}=\operatorname{dmatrix}(1$, num, 1, num +1$)$

$\mathrm{D}=\operatorname{dmatrix}(1,1,1$, num +1$)$;

for $(\mathrm{i}=1 ; \mathrm{i}<=$ num; $\mathrm{i}++)$

for $(j=1 ; j<=$ num $+1 ; j++)\{$

$\mathrm{f}[\mathrm{i}][\mathrm{j}]=\mathrm{g}[\mathrm{i}][\mathrm{j}]$; 


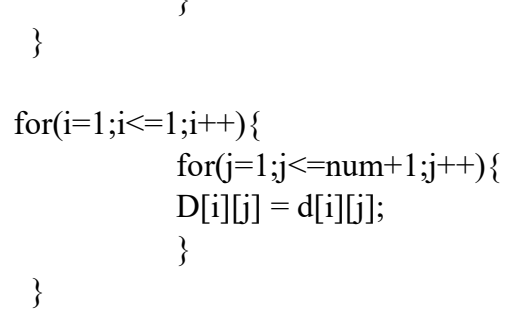

/* Memory release */

free_dmatrix (g, 1, num, 1, num +1$)$; free_dmatrix $(\mathrm{d}, 1,1,1, \mathrm{num}+1)$;

/* Memory allocation */

a_abs = dmatrix $(1, \mathrm{M}+2,1, \mathrm{M}+2)$;

$\mathrm{b}=$ dmatrix $(1$, num $+1,1$, num $)$;

$\mathrm{c}=$ dmatrix $(1$, num $+1,1$, num +1$)$;

$\mathrm{e}=$ dmatrix $(1$, num $+1,1$, num +1$)$;

g_abs = dmatrix $(1, \mathrm{M}+2,1, \mathrm{M}+2)$;

$\mathrm{sa}=\operatorname{dmatrix}(1$, num $+1,1$, num +1$)$;

inv_sa $=$ dmatrix $(1$, num $+1,1$, num +1$)$;

d_abs $=\operatorname{dmatrix}(1,1,1, \mathrm{M}+2)$;

/* Matrix setting: $\tilde{\mathrm{P}}_{\mathrm{i}}, \mathrm{Q}_{\mathrm{i}}, \mathrm{I}_{\mathrm{i}} * /$

upabs_matrix (a_abs,'A',fout);

dn matrix (b,'B',fout);

en_matrix(e,'I',fout);

/* Calculation: $\pi_{\mathrm{i}+1}=(1,0) \mathrm{F}_{1} \mathrm{~F}_{2} \mathrm{~F}_{3} \ldots \mathrm{F}_{\mathrm{i}-1} \mathrm{~F}_{\mathrm{i}} * /$

product1(c,b,f,e,sa,fout); /* $\mathrm{I}_{\mathrm{i}+1}-\mathrm{Q}_{\mathrm{i}} \mathrm{F}_{\mathrm{i}-1} * /$

gauss (sa,inv_sa,fout); $/ *\left(\mathrm{I}_{\mathrm{i}+1}-\mathrm{Q}_{\mathrm{i}} \mathrm{F}_{\mathrm{i}-1}\right)^{-1} * /$

pre_product2 (inv_sa,a_abs,g_abs,fout); $/ * \mathrm{~F}_{\mathrm{i}}=\left(\mathrm{I}_{\mathrm{i}+1}-\mathrm{Q}_{\mathrm{i}} \mathrm{F}_{\mathrm{i}-1}\right)^{-1} \mathrm{P}_{\mathrm{i}} * /$

pre_product3 (d_abs,D,g_abs, fout); $\quad / * \pi_{i+1}=(1,0) \mathrm{F}_{1} \mathrm{~F}_{2} \mathrm{~F}_{3} \ldots \mathrm{F}_{\mathrm{i}-1} \mathrm{~F}_{\mathrm{i}} * /$

/* Probability of resistance */ 
keisan(d_abs, prob, prob_d, P, *fout);

/* Print out */

fprintf (fout,"Probability is: ¥n",c);

fprintf (fout,"\%12.10f¥t”, keisan(d_abs,'P', fout));

fprintf (fout,"¥n");

* Memory release *

free_dmatrix(a_abs, 1, M+2, 1, M+2);

free dmatrix (b, 1 , num $+1,1$, num);

free_dmatrix (c, 1, num $+1,1$, num +1$)$;

free dmatrix (e, 1, num $+1,1$, num +1$)$

free_dmatrix(f, 1, num, 1, num +1 );

free_dmatrix (sa, 1, num $+1,1$, num +1 );

free dmatrix(inv sa, 1, num $+1,1$, num +1 );

free_dmatrix $(\mathrm{D}, \overline{1}, 1,1, \mathrm{num}+1)$;

* Calculation After Aggregation */

else

\{

/* Memory allocation */

f abs $=$ dmatrix $(1, M+2,1, M+2)$;

D_abs $=\operatorname{dmatrix}(1,1,1, \mathrm{M}+2)$;

For $(\mathrm{i}=1 ; \mathrm{i}<=\mathrm{M}+2 ; \mathrm{i}++)\{$

for $(j=1 ; j<=M+2 ; j++)\{$

f_abs[i][j] = g_abs[i] $[\mathrm{j}]$;

\}

for $(\mathrm{i}=1 ; \mathrm{i}<=1 ; \mathrm{i}++)$

for $(\mathrm{j}=1 ; \mathrm{j}<=\mathrm{M}+2 ; \mathrm{j}++)\{$ 
* Memory release */

free_dmatrix (g_abs, 1, M+2, 1, M+2);

free dmatrix (d abs, 1, 1, 1, M+2);

\section{/* Memory allocation */}

a_abs $=$ dmatrix $(1, \mathrm{M}+2,1, \mathrm{M}+2)$;

b_abs $=\operatorname{dmatrix}(1, \mathrm{M}+2,1, \mathrm{M}+2)$;

c_abs $=\operatorname{dmatrix}(1, \mathrm{M}+2,1, \mathrm{M}+2)$;

e_abs $=\operatorname{dmatrix}(1, \mathrm{M}+2,1, \mathrm{M}+2)$;

g_abs $=\operatorname{dmatrix}(1, \mathrm{M}+2,1, \mathrm{M}+2)$;

sa_abs $=\operatorname{dmatrix}(1, \mathrm{M}+2,1, \mathrm{M}+2)$;

inv_saabs = dmatrix $(1, \mathrm{M}+2,1, \mathrm{M}+2)$;

d_abs $=\operatorname{dmatrix}(1,1,1, \mathrm{M}+2)$;

/* Matrix setting: $\tilde{\mathrm{P}}_{\mathrm{i}}, \tilde{\mathrm{Q}}_{\mathrm{i}}, \tilde{\mathrm{I}}_{\mathrm{i}} * /$

upabs_matrix(a_abs,'A',fout);

dnabs matrix(b abs,'B',fout);

enabs_matrix(e_abs,'I',fout);

/*Calculation: $\tilde{\pi}_{\mathrm{i}+1}=(1,0) \quad \tilde{\mathrm{F}}_{1} \quad \tilde{\mathrm{F}}_{2} \quad \tilde{\mathrm{F}}_{3} \ldots \tilde{\mathrm{F}}_{\mathrm{i}-1} \quad \tilde{\mathrm{F}}_{\mathrm{i}} * /$

product1_abs(c_abs,b_abs,f_abs,e_abs,sa_abs,fout); $/ * \tilde{\mathrm{I}}_{\mathrm{i}+1}-\tilde{\mathrm{Q}}_{\mathrm{i}} \tilde{\mathrm{F}}_{\mathrm{i}-1} * /$ gauss_abs(sa_abs,inv_saabs,fout); $\left./ * \quad \overline{\mathrm{I}}_{\mathrm{i}+1}-\tilde{\mathrm{Q}}_{\mathrm{i}} \tilde{\mathrm{F}}_{\mathrm{i}-1}\right)^{-1 * /}$

product2_abs(inv_saabs,a_abs,g_abs,fout); $\quad / * \tilde{\mathrm{F}}_{\mathrm{i}}=\left(\tilde{\mathrm{I}}_{\mathrm{i}+1}-\tilde{\mathrm{Q}}_{\mathrm{i}} \tilde{\mathrm{F}}_{\mathrm{i}-1}\right)^{-1} \tilde{\mathrm{P}}_{\mathrm{i}} * /$ product3_abs(d_abs,D_abs,g_abs,fout); $/ * \tilde{\pi}_{\mathrm{i}+1}=(1,0) \tilde{\mathrm{F}}_{1} \tilde{\mathrm{F}}_{2} \tilde{\mathrm{F}}_{3} \ldots \tilde{\mathrm{F}}_{\mathrm{i}-1} \tilde{\mathrm{F}}_{\mathrm{i}} * /$

/* Probability of resistance */

keisan(d_abs, prob, prob_d, P, *fout);

/* Print out */ 
fprintf (fout,"Probability is : ¥n",c);

fprintf (fout,"\%12.10f¥t",prob);

fprintf (fout,"¥n");

/* Memory release */

free_dmatrix (a_abs, 1, M+2, 1, M+2);

free dmatrix (b abs, $1, \mathrm{M}+2,1, \mathrm{M}+2$ );

free_dmatrix (c_abs, 1, M+2, 1, M+2);

free_dmatrix (e_abs, $1, \mathrm{M}+2,1, \mathrm{M}+2)$

free dmatrix (f abs, 1, M+2, 1, M+2);

free_dmatrix (sa_abs, $1, \mathrm{M}+2,1, \mathrm{M}+2)$;

free dmatrix (inv saabs, 1, M+2, 1, M+2);

free_dmatrix (D_abs, 1, 1, 1, M+2);

\}

return (0);

/* Function for calculating matrix (exact): $\mathrm{P}_{\mathrm{i}} * /$ void up_matrix (double **a, char c, FILE *fout)

int $\mathrm{i}, \mathrm{j}$;

for $(\mathrm{i}=1 ; \mathrm{i}<=$ num $+1 ; \mathrm{i}++)$

\{

for $(j=1 ; j<=$ num $+2 ; j++)$

if $(I==j)\{$

$\mathrm{a}[\mathrm{i}][\mathrm{j}]=(($ num $-\mathrm{i}+1) * \lambda *(1-\gamma)) /((\lambda+\mu) *($ num $-\mathrm{i}+1)+(\alpha+\beta) *(\mathrm{i}-1))$

\}

else if $(i+1==\mathrm{j})$

$\mathrm{a}[\mathrm{i}][\mathrm{j}]=(($ num-i +1$) * \lambda * \gamma+(\mathrm{i}-1) * \alpha) /((\lambda+\mu) *($ num-i +1$)+(\alpha+\beta) *(\mathrm{i}-1))$; 


$$
\text { else }\{
$$

$\mathrm{a}[\mathrm{i}][\mathrm{j}]=0$;

\}

\}

/* Function for calculating matrix (exact): $\mathrm{Q}_{\mathrm{i}} * /$

void dn_matrix(double**b, char c, FILE *fout)

int $i, j$;

for $(\mathrm{i}=1 ; \mathrm{i}<=$ num $+1 ; \mathrm{i}++)$

for $(j=1 ; j<=$ num; $j++)$

\{

if $(\mathrm{i}==\mathrm{j})\{$

$b[i][j]=\left((\text { num-i }+1)^{*} \mu\right) /\left((\lambda+\mu)^{*}(\right.$ num $\left.-i+1)+(\alpha+\beta) *(i-1)\right) ;$ else if $(\mathrm{i}==\mathrm{j}+1)$ \{

$\mathrm{b}[\mathrm{i}][\mathrm{j}]=((\mathrm{i}-1) * \beta) /((\lambda+\mu) *($ num $-\mathrm{i}+1)+(\alpha+\beta) *(\mathrm{i}-1))$;

\}

else \{

$\mathrm{b}[\mathrm{i}][\mathrm{j}]=0$;

\}

\}

* Function for calculating matrix (aggregation) : $\tilde{\mathrm{P}}_{\mathrm{i}} * /$ void upabs matrix (double **a abs, char c, FILE *fout)

int $\mathrm{i}, \mathrm{j}$; 


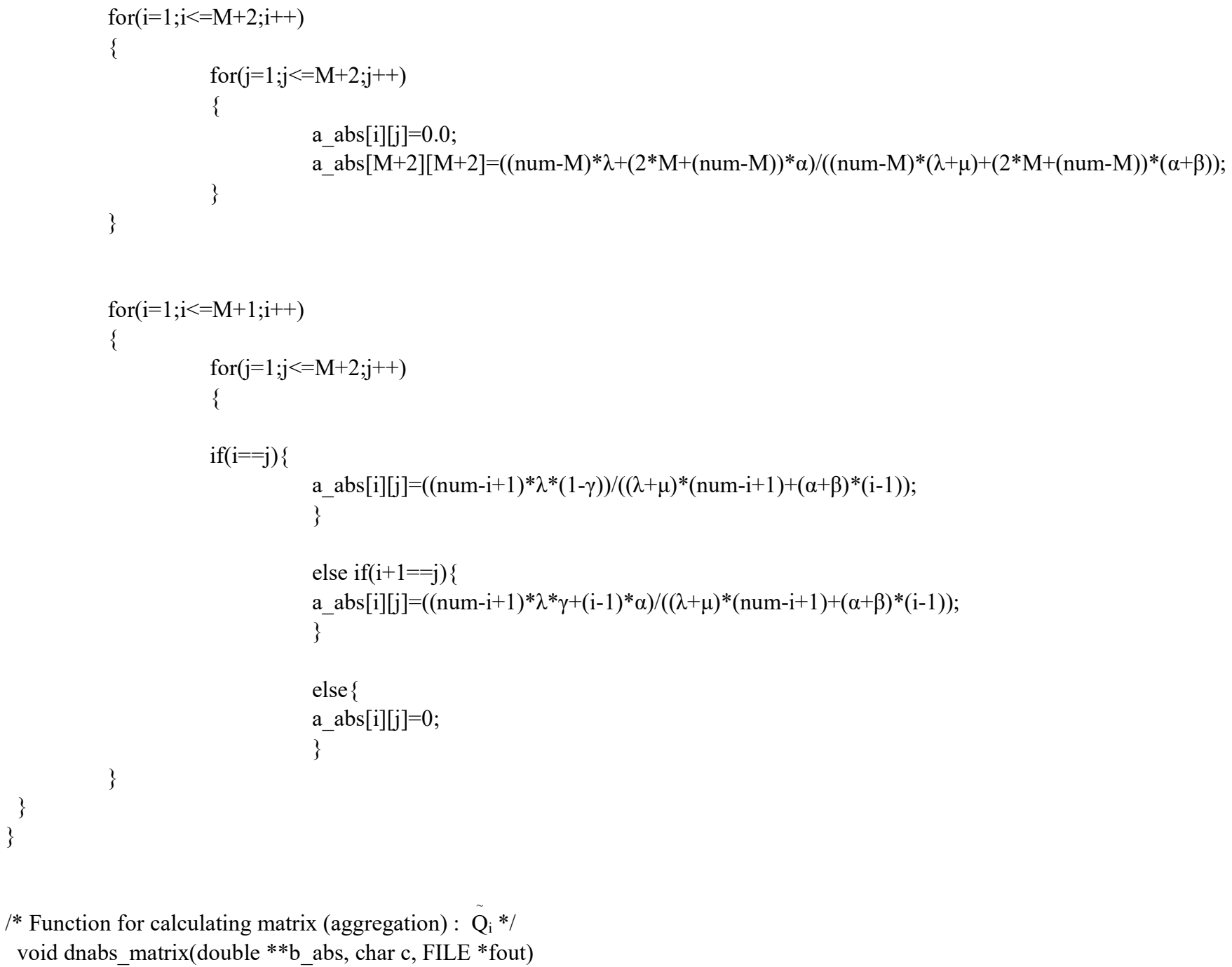


for $(\mathrm{i}=1 ; \mathrm{i}<=\mathrm{M}+2 ; \mathrm{i}++)$

\{

for $(\mathrm{j}=1 ; \mathrm{j}<=\mathrm{M}+2 ; \mathrm{j}++)$

\{

$\left\{\begin{array}{l}\text { if }(\text { num }<=46340) \\ \{ \\ \text { b } \operatorname{abs}[i][j]=0.0\end{array}\right.$

b_abs[i][j] $=0.0$;

b_abs[M+2][M+2] $=(($ num-M $) *($ num-M +1$) * \mu+(2 *($ num-M $) * M+($ num-M $) *($ num-M +1$)) * \beta) /((\lambda+\mu) *($ num-M $) *($ num-M +1$)+(\alpha+\beta) *(2 *($ num-

$\mathrm{M}+1) * \mathrm{M}+($ num-M)*(num-M+1)));

b_abs[M+2][M+1] $=(2 * \mathrm{M} * \beta) /((\lambda+\mu) *($ num-M $) *($ num-M +1$)+(\alpha+\beta) *(2 *($ num-M +1$) * \mathrm{M}+($ num-M $) *($ num $-\mathrm{M}+1)))$;

else

\{

b_abs[i][j] $=0.0$;

b abs[M+2][M+2] $=(($ num-M $) * \mu+(2 * \mathrm{M} *(1-1 /($ num-M +1$))+($ num-M $)) * \beta) /((\lambda+\mu) *($ num-M $)+(\alpha+\beta) *(2 * \mathrm{M}+($ num $-\mathrm{M})))$;

b_abs[M+2][M+1] $=(1 /($ num-M +1$)) *(2 * \beta * M) /((\lambda+\mu) *($ num $-M)+(\alpha+\beta) *(2 * M+($ num $-M)))$;

\}

for $(\mathrm{i}=1 ; \mathrm{i}<=\mathrm{M}+1 ; \mathrm{i}++)$

\{

for $(\mathrm{j}=1 ; \mathrm{j}<=\mathrm{M}+1 ; \mathrm{j}++)$

if $(\mathrm{i}==\mathrm{j})\{$

b_abs $[\mathrm{i}][\mathrm{j}]=(($ num-i+1)* $\mu) /((\lambda+\mu) *($ num $-\mathrm{i}+1)+(\alpha+\beta) *(\mathrm{i}-1))$;

else if $(i=-j+1)\{$

b abs $[\mathrm{i}][\mathrm{j}]=(\mathrm{j} * \beta) /((\lambda+\mu) *($ num-i -1$)+(\alpha+\beta) *(\mathrm{i}-1))$;

\} 


\section{else \{}

b_abs[i] $[\mathrm{j}]=0$;

\}

/* Function for calculating unit matrix (exact): $\mathrm{I}_{\mathrm{i}} * /$

void en_matrix(double **e, char c, FILE *fout)

int $\mathrm{i}, \mathrm{j}$;

for $(\mathrm{i}=1 ; \mathrm{i}<=$ num $+1 ; \mathrm{i}++)$

for $(j=1 ; j<=$ num $+1 ; j++)$

if $(\mathrm{i}==\mathrm{j})\{$

$\mathrm{e}[\mathrm{i}][\mathrm{j}]=1.0$;

\}

else \{

$\mathrm{e}[\mathrm{i}][\mathrm{j}]=0.0$;

\}

\}

\}

/* Function for calculating (exact): $\mathrm{I}_{\mathrm{i}+1}-\mathrm{Q}_{\mathrm{i}} \mathrm{F}_{\mathrm{i}-1}$ */

void product 1 (double ${ }^{* *} \mathrm{c}$, double $* *$ b, double $* *$ f, double $* *$ e, double $* *$ sa, FILE $*$ fout)

\{

int $\mathrm{i}, \mathrm{j}, \mathrm{k}$;

for $(\mathrm{i}=1 ; \mathrm{i}<=$ num $+1 ; \mathrm{i}++)$

\{

for $(j=1 ; j<=$ num $+1 ; j++)$ 
$\mathrm{c}[\mathrm{i}][\mathrm{j}]=0.0 ;$

for $(\mathrm{k}=1 ; \mathrm{k}<=$ num $; \mathrm{k}++)$

$$
\mathrm{c}[\mathrm{i}][\mathrm{j}]+=\mathrm{b}[\mathrm{i}][\mathrm{k}] * \mathrm{f}[\mathrm{k}][\mathrm{j}] \text {; }
$$

$\mathrm{sa}[\mathrm{i}][\mathrm{j}]=\mathrm{e}[\mathrm{i}][\mathrm{j}]-\mathrm{c}[\mathrm{i}][\mathrm{j}] ;$

\}

$/ *$ Function for calculating (exact) $: \mathrm{F}_{\mathrm{i}}=\left(\mathrm{I}_{\mathrm{i}+1}-\mathrm{Q}_{\mathrm{i}} \mathrm{F}_{\mathrm{i}-1}\right)^{-1} * /$

void gauss (double $* *$ sa, double $* *$ inv sa, FILE $*$ fout)

\{

int i, j, k,ip;

double alpha, tmp

double amax , eps $=\operatorname{pow}(10.0,-13.0) ; /^{*}$ eps $=10^{\wedge}\{-13\} * /$

for $(\mathrm{i}=1 ; \mathrm{i}<=$ num $+1 ; \mathrm{i}++)$

for $(j=1 ; j<=$ num $+1 ; j++)$$$
\{
$$$$
\text { if }(\mathrm{i}==\mathrm{j})\{
$$

inv_sa[i] $[\mathrm{j}]=1.0$;

\}

else \{

inv_sa[i] $[\mathrm{j}]=0.0$;

\} 


\section{for $(\mathrm{k}=1 ; \mathrm{k}<=$ num $+1 ; \mathrm{k}++)$}

\section{/ Pivoting */}

$\operatorname{amax}=\operatorname{fabs}(\mathrm{sa}[\mathrm{k}][\mathrm{k}])$

$\mathrm{ip}=\mathrm{k}$;

for $(\mathrm{i}=\mathrm{k}+1 ; \mathrm{i}<$ num $+1 ; \mathrm{i}++)$

$\operatorname{if}(\operatorname{fabs}(\mathrm{sa}[\mathrm{i}][\mathrm{k}])>\operatorname{amax})$

$\operatorname{amax}=\operatorname{fabs}(\mathrm{sa}[\mathrm{i}][\mathrm{k}]) ;$

$\mathrm{ip}=\mathrm{i}$

\}

/* Determination of regularity */

if( amax $<$ eps ) printf(" The matrix is not regular ¥n");

/* Exchanging rows */

if( ip != k)

\{

for $(j=1 ; j<=$ num $+1 ; j++)$

\{

tmp $=\mathrm{sa}[\mathrm{k}][\mathrm{j}]$;

$\mathrm{sa}[\mathrm{k}][\mathrm{j}]=\mathrm{sa}[\mathrm{ip}][\mathrm{j}]$

$\mathrm{sa}[\mathrm{ip}][\mathrm{j}]=\mathrm{tmp}$;

tmp $=$ inv_sa[k][j]

inv_sa[k][j] = inv_sa[ip][j];

inv_sa[ip][j] $=\mathrm{tmp}$;

\}

* Forward elimination */ alpha $=1.0 / \mathrm{sa}[\mathrm{k}][\mathrm{k}]$; 
for $(i=1 ; i<=$ num $+1 ; i++)$

\{

$\mathrm{sa}[\mathrm{k}][\mathrm{i}]=$ alpha* $\mathrm{sa}[\mathrm{k}][\mathrm{i}]$

inv_sa[k][i] = alpha*inv_sa[k][i];

\}

* pivot column $=0 * /$

for $(\mathrm{i}=1 ; \mathrm{i}<=$ num $+1 ; \mathrm{i}++)$

\{

if $(\mathrm{i} !=\mathrm{k})$

tmp $=\mathrm{sa}[\mathrm{i}][\mathrm{k}]$

for $(j=1 ; j<=$ num $+1 ; j++)$

\{

$\mathrm{sa}[\mathrm{i}][\mathrm{j}]=\mathrm{sa}[\mathrm{i}][\mathrm{j}]-\mathrm{tmp} * \mathrm{sa}[\mathrm{k}][\mathrm{j}]$

inv_sa[i][j] = inv_sa[i][j]-tmp*inv_sa[k][j];

\}

/* Function for calculating (exact) : $\mathrm{F}_{\mathrm{i}}=\left(\mathrm{I}_{\mathrm{i}+1}-\mathrm{Q}_{\mathrm{i}} \mathrm{F}_{\mathrm{i}-1}\right)^{-1} \mathrm{P}_{\mathrm{i}} * /$

void product2 (double **inv_sa, double $* *$ a,double $* *$ g, FILE $*$ fout)

int i, j, k;

for $(\mathrm{i}=1 ; \mathrm{i}<=$ num $+1 ; \mathrm{i}++)$

for $(j=1 ; j<=$ num $+2 ; j++)$

$$
\{
$$
$\mathrm{g}[\mathrm{i}][\mathrm{j}]=0.0$;

for $(\mathrm{k}=1 ; \mathrm{k}<=$ num $+1 ; \mathrm{k}++)$ 


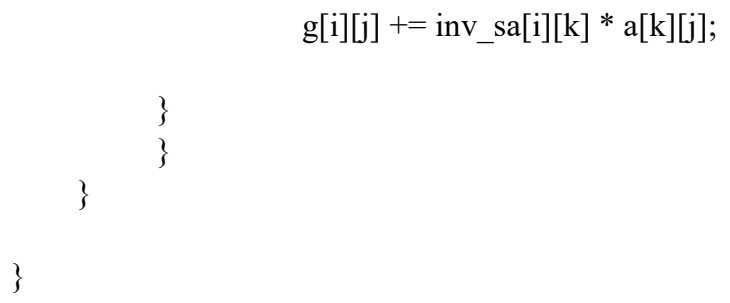

/* Function for calculating (exact) $: \pi_{\mathrm{i}+1}=(1,0) \mathrm{F}_{1} \mathrm{~F}_{2} \mathrm{~F}_{3} \ldots \mathrm{F}_{\mathrm{i}-1} \mathrm{~F}_{\mathrm{i}}$ */ void product3 (double $* *$ d, double $* * \mathrm{D}$, double $* *$ g, FILE * fout) \{

int $\mathrm{i}, \mathrm{j}, \mathrm{k}$;

for $(i=1 ; i<=1 ; i++)$

for $(j=1 ; j<=$ num $+2 ; j++)$

$$
\{\mathrm{d}[\mathrm{i}][\mathrm{j}]=0.0 \text {; }
$$$$
\mathrm{d}[\mathrm{i}][\mathrm{j}]+=\mathrm{D}[\mathrm{i}][\mathrm{k}] * \mathrm{~g}[\mathrm{k}][\mathrm{j}] ;
$$$$
\text { \} }
$$

\}

$/ *$ Function for calculating (exact) $: \pi_{2}=(1,0) \mathrm{F}_{1} * /$

void product4(double $* *$ dinit, double $* * \mathrm{~g}$, double $* *$ d, FILE *fout) \{

int $\mathrm{i}, \mathrm{j}, \mathrm{k}$; 
$\operatorname{dinit}[1][1]=1.0 ;$
$\operatorname{dinit}[1][2]=0.0 ;$

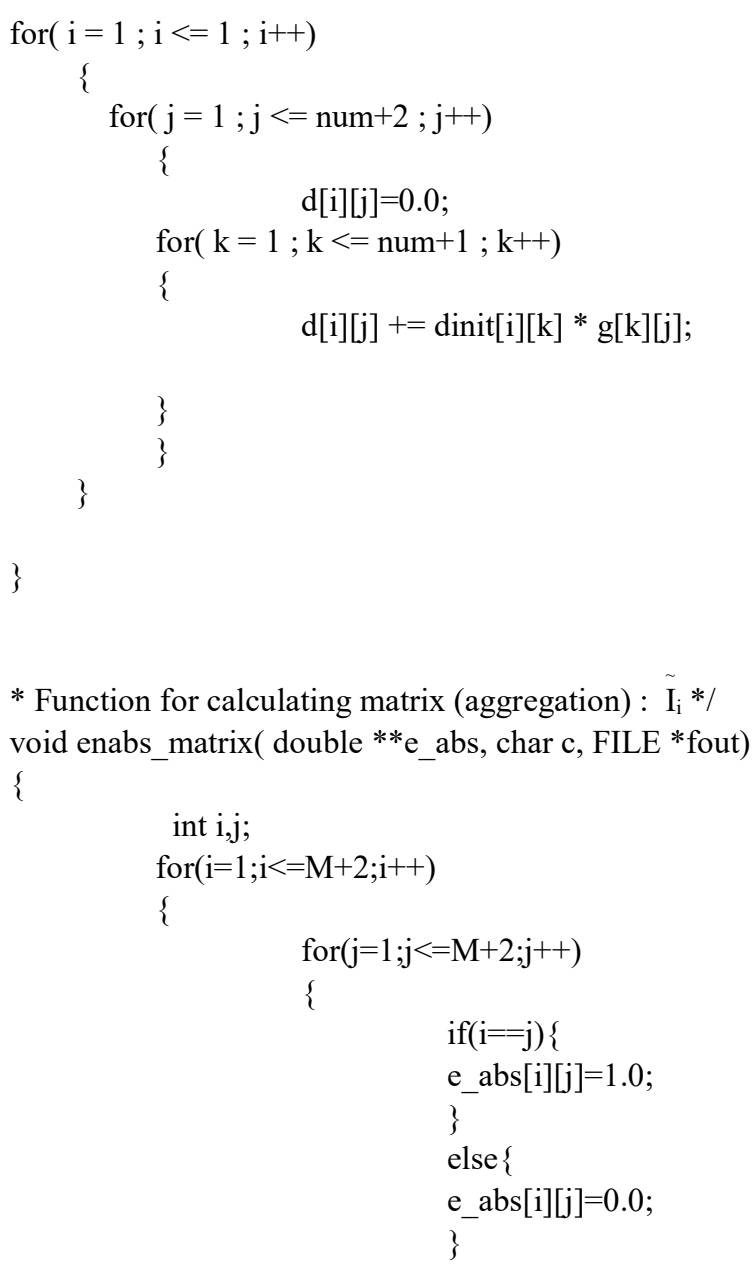

* Function for calculating matrix (aggregation) : $\tilde{\mathrm{I}}_{\mathrm{i}} * /$

void enabs_matrix (double $* *$ e_abs, char c, FILE *fout)

\{ 
/*Function for calculating (aggregation): $\tilde{\mathrm{I}}_{\mathrm{i}+1}-\tilde{\mathrm{Q}}_{\mathrm{i}} \tilde{\mathrm{F}}_{\mathrm{i}-1}$ */

void product1_abs(double ${ }^{* *}$ __abs, double $* *$ b_abs, double $* *$ f_abs, double $* *$ e_abs,double $* *$ sa_abs,FILE *fout)

int $\mathrm{i}, \mathrm{j}, \mathrm{k}$;

for $(\mathrm{i}=1 ; \mathrm{i}<=\mathrm{M}+2 ; \mathrm{i}++)$

for $(j=1 ; j<=M+2 ; j++)$

c abs $[\mathrm{i}][\mathrm{j}]=0.0$;

for $(\mathrm{k}=1 ; \mathrm{k}<=\mathrm{M}+2 ; \mathrm{k}++)$

abs[i] $[j]+=b \_a b s[i][k] * f$ abs $[k][j]$

sa_abs[i][j] = e_abs[i][j] - c_abs[i]][j];

(1)

/*Function for calculating (aggregation): $\left(\tilde{\mathrm{I}}_{\mathrm{i}+1}-\tilde{\mathrm{Q}}_{\mathrm{i}} \tilde{\mathrm{F}}_{\mathrm{i}-1}\right)^{-1 * /}$

void gauss_abs(double $* *$ sa_abs, double $* *$ inv_saabs,FILE $*$ fout $)$

int $\mathrm{i}, \mathrm{j}, \mathrm{k}, \mathrm{ip}$;

double alpha, tmp;

double amax eps $=\operatorname{pow}(10.0,-13.0) ; /^{*}$ eps $=10^{\wedge}\{-13\} * /$

for $(\mathrm{i}=1 ; \mathrm{i}<=\mathrm{M}+2 ; \mathrm{i}++)$

for $(\mathrm{j}=1 ; \mathrm{j}<=\mathrm{M}+2 ; \mathrm{j}++)$ 


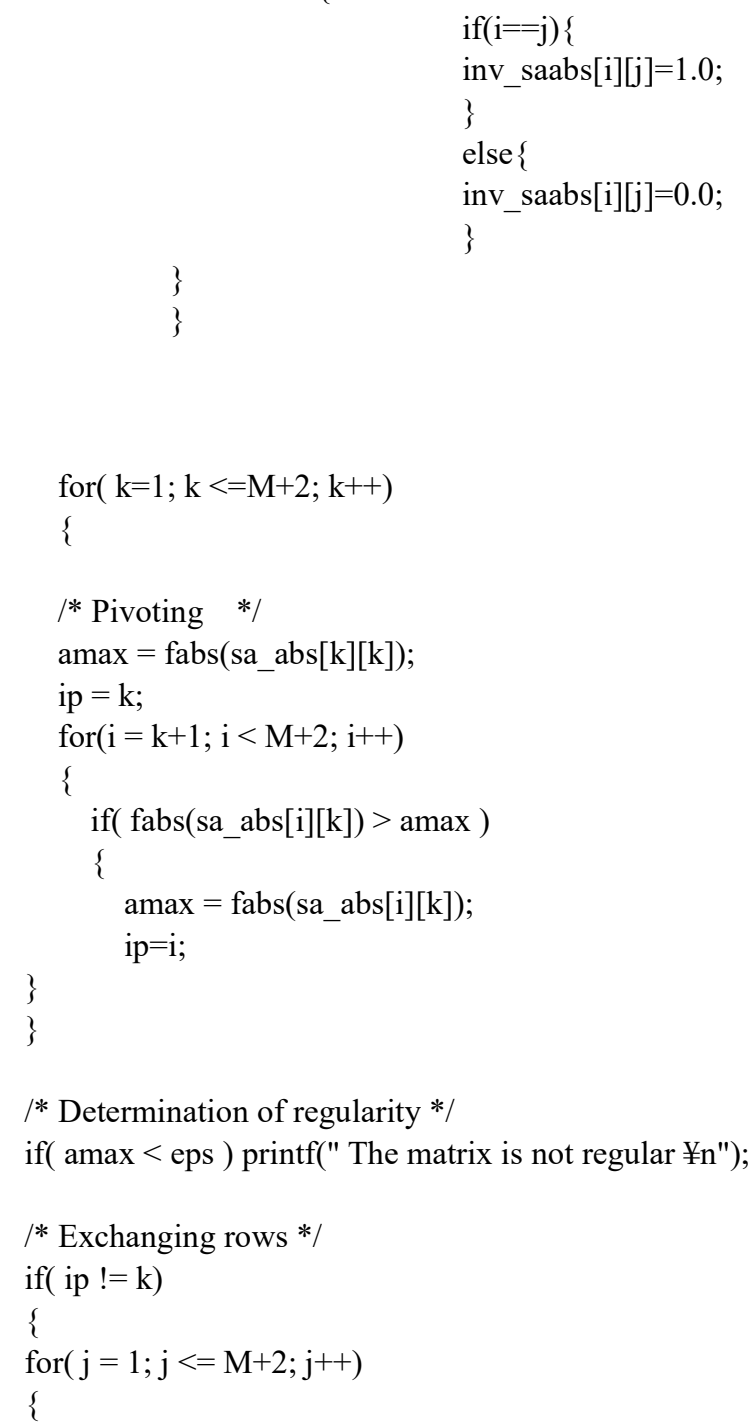


tmp $=$ sa $a b s[k][j] ;$

sa_abs[k][j] = sa_abs[ip][j];

sa $a b s[i p][j]=$ tmp;

tmp $=$ inv_saabs[k][j];

inv_saabs[k][j] = inv_saabs[ip][j];

inv $\operatorname{saabs}[i p][j]=\mathrm{tmp}$;

\}

/* Forward elimination *

alpha $=1.0 /$ sa $_{-}$abs $[\mathrm{k}][\mathrm{k}]$;

for $(\mathrm{i}=1 ; \mathrm{i}<=\mathrm{M}+2 ; \overline{\mathrm{i}}++$ )

\{

sa_abs[k][i] = alpha*sa_abs[k][i];

inv_saabs[k][i] = alpha*inv_saabs[k][i];

* Pivot columns $=0$ *

for $(\mathrm{i}=1 ; \mathrm{i}<=\mathrm{M}+2 ; \mathrm{i}++)$

if $(\mathrm{i} !=\mathrm{k})$

tmp = sa_abs $[\mathrm{i}][\mathrm{k}]$;

for $(j=1 ; j<=M+2 ; j++)$

sa abs[i][j] = sa abs[i][j]-tmp*sa abs[k][j];

inv_saabs[i][j] = inv_saabs[i][j]-tmp*inv_saabs[k][j];

\}

\}

/* Function for calculating (aggregation) $: \tilde{\mathrm{F}}_{\mathrm{i}}=\left(\tilde{\mathrm{I}}_{\mathrm{i}+1}-\tilde{\mathrm{Q}}_{\mathrm{i}} \tilde{\mathrm{F}}_{\mathrm{i}-1}\right)^{-1} \tilde{\mathrm{P}}_{\mathrm{i}} * /$ 
void product2_abs( double **inv_saabs, double **a_abs,double **g_abs,FILE *fout) \{

int $\mathrm{i}, \mathrm{j}, \mathrm{k}$;

for $(\mathrm{i}=1 ; \mathrm{i}<=\mathrm{M}+2 ; \mathrm{i}++)$

for $(\mathrm{j}=1 ; \mathrm{j}<=\mathrm{M}+2 ; \mathrm{j}++)$

$/ *$ Function for calculating (aggregation) : $\tilde{\pi}_{\mathrm{i}+1}=(1,0) \quad \tilde{\mathrm{F}}_{1} \tilde{\mathrm{F}}_{2} \quad \tilde{\mathrm{F}}_{3} \ldots \tilde{\mathrm{F}}_{\mathrm{i}-1} \tilde{\mathrm{F}}_{\mathrm{i}} * /$

void product3 abs( double ${ }^{* *} \mathrm{~d}$ abs, double ${ }^{* *} \mathrm{D}$ abs, double ${ }^{* *} \mathrm{~g}$ abs,FILE *fout)

\{

int $\mathrm{i}, \mathrm{j}, \mathrm{k}$;

for $(i=1 ; i<=1 ; i++)$

\{

for $(\mathrm{j}=1 ; \mathrm{j}<=\mathrm{M}+2 ; \mathrm{j}++)$

d abs $[\mathrm{i}][\mathrm{j}]=0.0$;

for $(\mathrm{k}=1 ; \mathrm{k}<=\mathrm{M}+2 ; \mathrm{k}++)$

\{

d_abs[i][j] += D_abs[i][k]*g_abs[k][j]; 
/* Function for calculating (aggregation point) $: \tilde{\mathrm{F}}_{\mathrm{i}}=\left(\tilde{\mathrm{I}}_{\mathrm{i}+1}-\tilde{\mathrm{Q}}_{\mathrm{i}} \tilde{\mathrm{F}}_{\mathrm{i}-1}\right)^{-1} \tilde{\mathrm{P}}_{\mathrm{i}} * /$

void pre_product2( double **inv sa, double $* *$ a abs,double $* *$ g abs,FILE *fout)

int $\mathrm{i}, \mathrm{j}, \mathrm{k}$;

for $(\mathrm{i}=1 ; \mathrm{i}<=$ num $+1 ; \mathrm{i}++)$

\{

for $(\mathrm{j}=1 ; \mathrm{j}<=\mathrm{M}+2 ; \mathrm{j}++)$

for $(\mathrm{k}=1 ; \mathrm{k}<=$ num $+1 ; \mathrm{k}++)$

g_abs[i][j] += inv_sa[i][k]*a_abs[k][j];

\}

\}

* Function for calculating (aggregation point) : $\tilde{\pi}_{\mathrm{i}+1}=(1,0) \quad \tilde{\mathrm{F}}_{1} \tilde{\mathrm{F}}_{2} \tilde{\mathrm{F}}_{3} \ldots \tilde{\mathrm{F}}_{\mathrm{i}-1} \quad \tilde{\mathrm{F}}_{\mathrm{i}}^{*} /$ void pre_product3( double ${ }^{* *}$ d_abs, double $* * D$, double ${ }^{* *}$ g_abs,FILE *fout) \{

int $\mathrm{i}, \mathrm{j}, \mathrm{k}$;

for $(i=1 ; i<=1 ; i++)$

\{ 


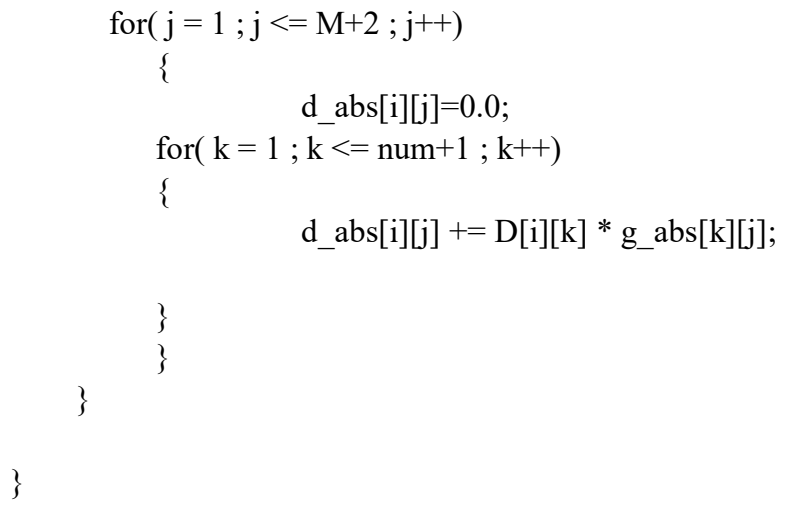

/* Function for calculating probability */

double keisan (double ${ }^{* *} \mathrm{x}$, double *prob, double *prob d, char c, FILE *fout)

int $i, j$

if (num $<=\mathrm{M}+1)\{$

for $(\mathrm{i}=1 ; \mathrm{i}<=1 ; \mathrm{i}++)$

\{

for $(j=1 ; j<=n u m+2 ; j++)\{$

sum $1=\operatorname{sum} 1+x[i][j]$;

\}

for $(\mathrm{i}=1 ; \mathrm{i}<=1 ; \mathrm{i}++)$

for $(j=2 ; j<=$ num $+2 ; j++)$

$\operatorname{sum} 2=\operatorname{sum} 2+x[i][j]$;

\} 
else \{

for $(\mathrm{i}=1 ; \mathrm{i}<=1 ; \mathrm{i}++)$

\{

for $(\mathrm{j}=1 ; \mathrm{j}<=\mathrm{M}+2 ; \mathrm{j}++)$

sum $1=\operatorname{sum} 1+x[i][j]$;

\}

for $(\mathrm{i}=1 ; \mathrm{i}<=1 ; \mathrm{i}++)$

for $(j=2 ; j<=M+2 ; j++)$

$\operatorname{sum} 2=\operatorname{sum} 2+x[i][j]$

\}

prob=sum $2 /$ sum $1 ;$

prob $d=$ prob $d+$ prob;

/* Memory allocation */

double **dmatrix(int nr1, int nr2, int nl1, int n12)

int i, nrow, ncol;

double **a;

nrow $=\mathrm{nr} 2-\mathrm{nr} 1+1 ;$

$\mathrm{ncol}=\mathrm{nl} 2-\mathrm{nll}+1$

if $\left(\left(\mathrm{a}=\left(\right.\right.\right.$ double $\left.^{* *}\right) \operatorname{malloc}($ nrow*sizeof $($ double $\left.*))\right)==$ NULL $)$ 


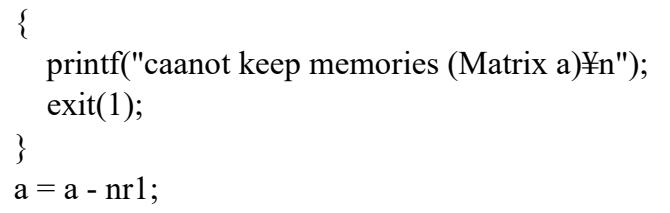

for $(\mathrm{i}=\mathrm{nr} 1 ; \mathrm{i}<=\mathrm{nr} 2 ; \mathrm{i}++) \mathrm{a}[\mathrm{i}]=($ double $*) \operatorname{malloc}($ ncol*sizeof $($ double $))$; for $(\mathrm{i}=\mathrm{nr} 1 ; \mathrm{i}<=\mathrm{nr} 2 ; \mathrm{i}++) \mathrm{a}[\mathrm{i}]=\mathrm{a}[\mathrm{i}]-\mathrm{nl}$;

return(a);

* Memory release */

void free_dmatrix(double **a, int nr1, int nr2, int nl1, int nl2)

int $\mathrm{i}$;

for $(\mathrm{i}=\mathrm{nr} 1 ; \mathrm{i}<=\mathrm{nr} 2 ; \mathrm{i}++)$ free $((\operatorname{void} *)(\mathrm{a}[\mathrm{i}]+\mathrm{nl} 1))$; free $((\operatorname{void} *)(\mathrm{a}+\mathrm{nr} 1))$; 